\title{
Weekly administration of paclitaxel and carboplatin with concurrent thoracic radiation in previously untreated elderly patients with locally advanced non-small-cell lung cancer: A case series of 20 patients
}

\author{
JUMPEI TAKESHITA ${ }^{1}$, KATSUHIRO MASAGO ${ }^{1}$, SHIRO FUJITA ${ }^{1}$, AKITO HATA ${ }^{1}$, REIKO KAJI ${ }^{1}$, \\ TAKAHISA KAWAMURA ${ }^{2}$, KOJI TAMAI ${ }^{2}$, TAKESHI MATSUMOTO ${ }^{2}$, KAZUMA NAGATA $^{2}$, KYOKO OTSUKA $^{2}$, \\ ATSUSHI NAKAGAWA ${ }^{2}$, KOJIRO OTSUKA ${ }^{2}$, KEISUKE TOMII ${ }^{2}$, TAKASHI SHINTANI ${ }^{3}$, \\ KENJI TAKAYAMA ${ }^{3}$, MASAKI KOKUBO ${ }^{4}$ and NOBUYUKI KATAKAMI ${ }^{1}$ \\ ${ }^{1}$ Division of Integrated Oncology, Institute of Biomedical Research and Innovation; ${ }^{2}$ Department of Respiratory Medicine, \\ Kobe City Medical Center General Hospital; ${ }^{3}$ Division of Radiation Oncology, \\ Institute of Biomedical Research and Innovation; ${ }^{4}$ Department of Radiation Oncology, \\ Kobe City Medical Center General Hospital, Kobe, Hyogo, Japan
}

Received December 16, 2013; Accepted January 23, 2014

DOI: $10.3892 / \mathrm{mco} .2014 .249$

\begin{abstract}
Elderly patients with stage III non-small-cell lung cancer (NSCLC) are frequently underrepresented in clinical trials that evaluate chemoradiotherapy, due to their poor functional status, coexisting illnesses and limited life expectancy. The Japan Clinical Oncology Group 0301 trial (JCOG0301) was the first study to demonstrate that thoracic radiation therapy (TRT) with daily low-dose carboplatin may improve the outcome of elderly patients with stage III NSCLC. However, the efficacy and safety profiles of chemoradiotherapy, including platinum doublets, have not been clearly determined in this patient population. We retrospectively assessed the efficacy and toxicity of weekly paclitaxel in combination with carboplatin and concurrent TRT in patients aged $\geq 75$ years with previously untreated locally advanced NSCLC. Between February, 2004 and July, 2013, we collected the data of 20 patients treated with weekly paclitaxel and carboplatin for 6 weeks and concurrent TRT. The objective response rate was $90 \%$, the disease control rate was $95 \%$, the median progression-free survival was 8.63 months [95\% confidence interval (CI): 5.7-16.7] and the median overall survival (OS) was 16.1 months (95\% CI: 10.7-41.6). There were no grade 4 hematological or non-hematological toxicities and no reported treatment-related deaths. Therefore, platinum doublet
\end{abstract}

Correspondence to: Dr Jumpei Takeshita, Division of Integrated Oncology, Institute of Biomedical Research and Innovation, 2-2 Minatojima-Minamimachi, Chuo-ku, Kobe, Hyogo 650-0047, Japan

E-mail: jumpeinr2tfm3@fbri.org

Key words: locally advanced non-small cell lung cancer, platinum doublet, chemoradiotherapy, elderly patient, paclitaxel therapy in combination with TRT did not provide a clinically significant survival benefit in our population of elderly patients with locally advanced NSCLC. However, the present study demonstrated the good feasibility and safety of this regimen. Further prospective clinical trials are required to evaluate the efficacy and safety of platinum doublet with TRT in elderly patients.

\section{Introduction}

Lung cancer remains the leading cause of cancer-related mortality worldwide (1). This disease is more common among the elderly, with $>2 / 3$ of lung cancer cases occurring in individuals aged $\geq 65$ years and a median age at diagnosis of 70 years (2). The majority of lung cancer patients present with unresectable disease and are candidates for thoracic radiation therapy (TRT) and/or chemotherapy. Previously randomized clinical trials revealed a survival benefit for concurrent vs. sequential chemoradiotherapy $(3,4)$ and for sequential chemoradiotherapy vs. radiotherapy alone for patients with stage III non-small-cell lung cancer (NSCLC) $(5,6)$.

Concurrent chemoradiotherapy with platinum-based doublet chemotherapy is currently considered as the standard treatment for patients with inoperable stage III NSCLC. However, data from the Surveillance Epidemiology and End Results database reveal that the majority of elderly patients do not receive combined modality treatment (7). This finding may reflect the uncertainty regarding concurrent chemoradiotherapy as a treatment for elderly patients with locally advanced NSCLC.

In elderly patients, the use of concurrent chemoradiotherapy is often limited by poor functional status, coexisting illnesses, limited life expectancy and the physicians' concerns regarding toxicity and the effect of the treatment on the quality of life (QOL) of the patients. In addition, the number of available 
A

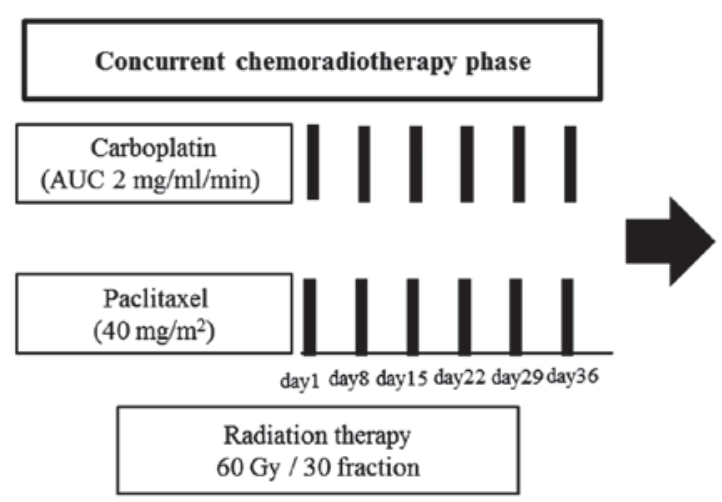

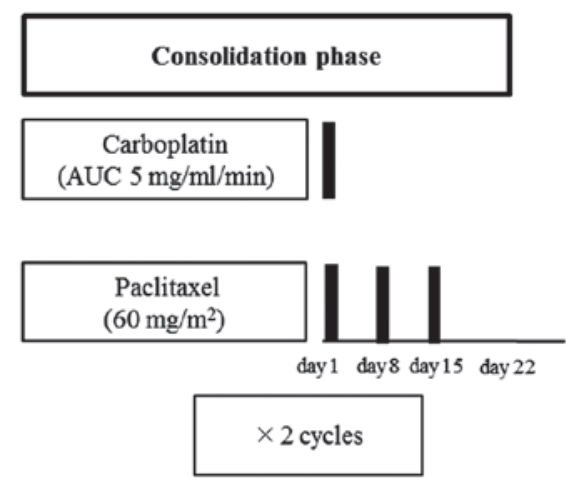

Figure 1. Chemotherapy schedule. (A) Concurrent chemoradiotherapy phase: paclitaxel ( $\left.40 \mathrm{mg} / \mathrm{m}^{2}\right)$ and carboplatin area under the curve (AUC) at $2 \mathrm{mg} / \mathrm{ml} / \mathrm{min}$ were administered concomitantly on the first day of the week. (B) Consolidation phase: weekly paclitaxel ( $\left.70 \mathrm{mg} / \mathrm{m}^{2}\right)$ for 3 of 4 weeks with carboplatin (AUC, $5 \mathrm{mg} / \mathrm{ml} / \mathrm{min}$ ) on day 1 of each 4 -week cycle.

clinical trials designed to specifically evaluate the treatment of elderly patients with stage III NSCLC is limited. Therefore, it is crucial to establish an effective and feasible chemoradiotherapy regimen for elderly patients with stage III NSCLC.

The Japan Clinical Oncology Group 0301 trial (JCOG0301) recently reported a significant survival advantage for elderly patients who received chemoradiotherapy (daily low-dose carboplatin plus radiotherapy) for locally advanced NSCLC (8). That trial provided reasonably strong evidence that single-agent carboplatin-based chemoradiotherapy is well-tolerated by elderly patients with locally advanced NSCLC and may achieve improved survival rates compared to radiotherapy alone. However, it has not been determined whether a carboplatin-based doublet regimen with TRT is feasible for elderly patients with locally advanced NSCLC.

The present study focused on the effectiveness of weekly paclitaxel in combination with carboplatin and concurrent TRT, since the efficacy and safety of this regimen in younger patients with locally advanced NSCLC was confirmed by phase III trials (9). The aim of our retrospective analysis was to assess the anticancer effect and toxicity of weekly paclitaxel and carboplatin with concurrent TRT in patients aged $\geq 75$ years with previously untreated locally advanced NSCLC.

\section{Patients and methods}

Patients. This retrospective study was performed at the Institute of Biomedical Research and Innovation and the Kobe City Medical Center General Hospital, Hyogo, Japan. The data from 20 consecutive patients, aged $\geq 75$ years, who were treated with weekly paclitaxel and carboplatin for 6 weeks, plus TRT (60 Gy) for locally advanced unresectable NSCLC (stage IIIA or IIIB) between February, 2004 and July, 2013 were retrospectively evaluated. This study was approved by the Institutional Review Board of the two hospitals.

The diagnosis of locally advanced unresectable stage III NSCLC was confirmed by a multidisciplinary council consisting of radiologists, radiation oncologists and medical oncologists prior to the initiation of the treatment. All the patients were diagnosed with NSCLC and the diagnosis was histopathologically confirmed. Tumour staging was performed by chest radiography, computed tomography (CT) scan or magnetic resonance imaging of the head, CT scan of the chest and the abdomen, CT scan or ultrasonography of the abdomen and bone scintigraphy or fluorodeoxyglucose positron emission tomography and CT scan. The patients were staged according to the tumour-node-metastasis (TNM) classification (10). An Eastern Cooperative Oncology Group (ECOG) performance status (PS) of 0-2 was required for inclusion in this study (11).

Radiotherapy. Radiotherapy for all the patients consisted of $60 \mathrm{~Gy}$ administered as 30 fractions over 6 weeks. A total of $40 \mathrm{~Gy}$ was delivered with 6-10 MV photons using anterior-posterior opposed fields that included the primary tumour, metastatic lymph nodes and regional nodes. A booster dose of $20 \mathrm{~Gy}$ was delivered to the primary tumour and the metastatic lymph nodes with off-cord fields, for a total dose of 60 Gy. Three-dimensional CT simulation was used for treatment planning. The clinical target volume included the gross tumour volume, including the primary tumour and metastatic nodes $(>1 \mathrm{~cm}$ at the shortest dimension), plus a $0.5-\mathrm{cm}$ margin. The regional nodes, excluding the contralateral hilar nodes, were also included in the clinical target volume. The planning target volumes for the primary tumour, metastatic lymph nodes and regional nodes were calculated as the clinical target volume plus adequate margins (typically $0.5-1.0 \mathrm{~cm}$ laterally and $1.0-2.0 \mathrm{~cm}$ craniocaudally).

The treatment plan was designed not to exceed the maximum doses tolerated by intrathoracic structures, such as the lung, spinal cord and heart. The spinal cord was excluded from the boost field by the oblique opposing method. If the dose of the spinal cord exceeded 40 Gy when planning the treatment, the minimum dose of the planning target volumes was modified and the dose to the spinal cord was restricted within $40 \mathrm{~Gy}$. Patients were excluded from this study if the initial radiation field exceeded half of the ipsilateral lung. If $\geq$ grade 3 oesophagitis occurred and the physician decided that the RT could not be continued, the treatment was suspended and reinitiated following recovery of the oesophagitis to sgrade 2 .

Chemotherapy. The treatment schedule is shown in Fig. 1. During radiotherapy, paclitaxel $\left(40 \mathrm{mg} / \mathrm{m}^{2}\right)$ and carboplatin 
area under the curve (AUC) at $2 \mathrm{mg} / \mathrm{ml} / \mathrm{min}$ were administered concomitantly on the first day of the week. The consolidation phase chemotherapy, initiated 3-4 weeks after the concurrent chemoradiotherapy, was administered in 2 cycles. The consolidation chemotherapy consisted of 3 weekly cycles of paclitaxel $\left(200 \mathrm{mg} / \mathrm{m}^{2}\right.$ administered over $\left.3 \mathrm{~h}\right)$ followed by carboplatin (AUC, $5 \mathrm{mg} / \mathrm{ml} / \mathrm{min}$ on day 1) or paclitaxel $\left(70 \mathrm{mg} / \mathrm{m}^{2}\right.$ ) weekly for 3 of 4 weeks with carboplatin (AUC, $5 \mathrm{mg} / \mathrm{ml} / \mathrm{min}$ ) on day 1 of each 4 -week cycle. During radiation treatment, paclitaxel and carboplatin administration was suspended if grade 4 hematological toxicity occurred and chemotherapy was reinitiated following recovery to $\leq$ grade 3 . A maximum of one dose level reduction was permitted per patient in the consolidation phase. The dose of carboplatin was reduced to achieve an AUC of $4 \mathrm{mg} / \mathrm{ml} / \mathrm{min}$ and the dose of paclitaxel was reduced to $175 \mathrm{mg} / \mathrm{m}^{2}$, or the weekly paclitaxel dose was reduced to $60 \mathrm{mg} / \mathrm{m}^{2}$. Both paclitaxel and carboplatin were reduced by one dose level if grade 4 hematological or $\geq$ grade 3 non-hematological toxicity occurred and the physician decided that chemotherapy should be discontinued.

Treatment and toxicity evaluation. The treatment efficacy and toxicity were assessed in all the treated patients. The patients were assessed for response by CT scans within 8 weeks of completing treatment. Following treatment completion, chest radiographs were obtained monthly and thoracic $\mathrm{CT}$ scans every 6 months. The patients underwent follow-up monthly for 1 year and at least every 3 months thereafter.

The treatment response evaluation was performed according to the Response Evaluation Criteria in Solid Tumours, version 1.09 (12), based only on the longest diameter of all the lesions as follows: Complete response (CR), disappearance of all the lesions; partial response (PR), $\geq 30 \%$ reduction of the sum of the longest diameters of all the lesions, referring to the sum of baseline longest diameters; progressive disease (PD), $\geq 20 \%$ increase in the sum of the longest diameters of the target lesions, referring to the smallest sum of the longest diameters recorded since the initiation of the treatment or the appearance of one or more new lesions; stable disease (SD), neither sufficient lesion shrinkage to qualify for PR, nor sufficient lesion growth to qualify for PD, referring to the smallest sum of the longest diameters since the initiation of the treatment. The toxicity was evaluated in accordance with the National Cancer Institute Common Toxicity Criteria 4.0.3 (13).

Statistical analysis. Median overall survival (OS) was defined as the time from the initiation of the treatment to death from any cause or the last follow-up. The patients who remained alive were evaluated at the date of the last follow-up. Progression-free survival (PFS) was defined as the time from the initiation of the treatment to disease progression (local recurrence and/or distant metastasis) or death. The respective contribution of local and distant progression to PFS and the rate of implementation of chemotherapy were estimated. The median follow-up time was calculated with the reverse Kaplan-Meier method and the Kaplan-Meier method was used for survival analysis. JMP software, version 9.0.0 (SAS Institute, Cary, NC, USA), was used for statistical analysis.
Table I. Patient characteristics $(\mathrm{n}=20)$.

\begin{tabular}{lr}
\hline Characteristics & Patients $(\%)$ \\
\hline Median age, years (range) & $78(75-86)$ \\
Gender & \\
Male & $17(85)$ \\
Female & $3(15)$ \\
Histology & \\
Adenocarcinoma & $8(40)$ \\
Squamous cell carcinoma & $12(60)$ \\
ECOG performance status & \\
0 & $2(10)$ \\
1 & $15(75)$ \\
2 & $3(15)$ \\
Disease stage & \\
IIIA & $9(45)$ \\
IIIB & $11(55)$ \\
Tumour stage & \\
1 & $2(10)$ \\
2 & $7(35)$ \\
3 & $4(20)$ \\
4 & $7(35)$ \\
Nodal stage & \\
1 & $2(10)$ \\
2 & $12(60)$ \\
3 & $6(30)$ \\
Comorbidity & \\
Hypertension & $5(25)$ \\
Diabetes & $2(10)$ \\
Cerebrovascular disease & $2(10)$ \\
Arrhythmia & $1(5)$ \\
Ischemic heart disease & $2(10)$ \\
Negoking history & \\
Positive & $18(90)$ \\
&
\end{tabular}

ECOG, Eastern Cooperative Oncology Group.

\section{Results}

Patient characteristics. Between February, 2004 and July, 2013, the data of 20 patients who were treated with weekly paclitaxel and carboplatin for 6 weeks plus 60 Gy TRT, were collected at the two abovementioned institutions. The median follow-up for censored cases was 21.1 months (interquartile range, 16.0-21.6). The pretreatment characteristics of the patients are summarized in Table I. The median age of the patients was 78 years, $85 \%$ of the patients were men, $90 \%$ had a history of smoking and $85 \%$ had an ECOG PS of 0-1. A total of $45 \%$ of the patients had stage IIIA and the remaining 55\% had stage IIIB disease. The reported comorbidities, listed by decreasing frequency, were hypertension $(25 \%)$, diabetes $(10 \%)$, cerebrovascular disease $(10 \%)$, ischemic heart disease (10\%) and arrhythmia (5\%). 
Table II. Chemotherapy administered.

\begin{tabular}{lcc}
\hline Chemotherapy & Cycles & Patients $(\%)$ \\
\hline Concurrent & 3 & $1(5)$ \\
& 4 & $1(5)$ \\
& 5 & $6(30)$ \\
Consolidation & 6 & $12(60)$ \\
& 0 & $6(30)$ \\
& 1 & $2(10)$ \\
& 2 & $12(60)$ \\
\hline
\end{tabular}

Treatment. The compliance to the protocol was considered as acceptable. The status of chemotherapy implementation is shown in Table II . During the concurrent phase, $60 \%$ of the patients received 6 weekly cycles of chemotherapy. In the consolidation phase, $60 \%$ of the patients received the two scheduled courses of therapy. All the patients completed TRT with a total dose of $60 \mathrm{~Gy}$.

Efficacy. In total, 1 patient achieved a CR, 17 achieved a PR, 1 had SD and 1 had PD (Table III). The objective response rate (ORR) was $90 \%$ and the disease control rate (DCR) was 95\%. The PFS and OS of the patients who were included in the trial are shown in Fig. 2. The median PFS was 8.6 months [95\% confidence interval (CI): 5.7-16.7] and the median OS was 16.1 months (95\% CI: 10.7-41.6).

Toxicity. The treatment-related adverse events are shown in Table IV. There were no reported grade 4 hematological and non-hematological toxicities. Grade 3 leukopenia occurred in $8(40 \%)$ and grade 3 neutropenia in 4 patients (20\%). Grade 2 pneumonitis occurred in 3 patients $(15 \%)$ on day 17 and at 3 and 7 months following treatment. All the cases required steroid therapy (prednisolone $20-40 \mathrm{mg} /$ day). Grade 2 oesophagitis occurred in 5 patients $(25 \%)$. There were no reported treatment-related deaths.

First site of disease progression. A total of 3 patients (15\%) exhibited local relapse, $9(45 \%)$ had distant metastasis and $2(10 \%)$ had both. Overall, 16 patients $(80 \%)$ exhibited disease progression and $17(85 \%)$ succumbed to the disease during the analysis, with 14 deaths due to the primary disease and 1 due to acute myocardial infarction at 379 days after treatment.

Post-treatment. A total of 8 patients (40\%) received second-line therapy (pemetrexed, 2; docetaxel, 1 ; gemcitabine, 1 ; vinorelbine, 1; S1, 1; gefitinib, 1; and erlotinib, 1). All the patients received palliative care and palliative radiation therapy as required.

\section{Discussion}

The present study retrospectively assessed the anticancer effect and toxicity of weekly paclitaxel in combination with carboplatin and concurrent TRT in previously untreated elderly patients (aged $\geq 75$ years) with locally advanced NSCLC. According to our results, the ORR was $90 \%$ and the DCR was $95 \%$, which were improved compared to those
Table III. Objective response.

\begin{tabular}{lc} 
Type of response & Patients $(\%)$ \\
\hline Complete & $1(5)$ \\
Partial & $17(85)$ \\
Stable disease & $1(5)$ \\
Progressive disease & $1(5)$ \\
Objective response rate & $18(90)$ \\
Disease control rate & $19(95)$
\end{tabular}

Table IV. Adverse events reported during the entire course of the treatment.

\begin{tabular}{lccc}
\hline & \multicolumn{3}{c}{ Toxicity (\%) } \\
\cline { 2 - 4 } Adverse event & Grade 2 & Grade 3 & Grade 4 \\
\hline Leukopenia & 8 & 8 & 0 \\
Neutropenia & 7 & 4 & 0 \\
Anaemia & 1 & 0 & 0 \\
Thrombocytopenia & 0 & 0 & 0 \\
Pneumonitis & 3 & 0 & 0 \\
Esophagitis & 5 & 0 & 0 \\
Pleural effusion & & & \\
(non-malignant) & 0 & 0 & 0 \\
Sensory neuropathy & 0 & 0 & 0 \\
Vomiting & 0 & 0 & 0 \\
Diarrhea & 0 & 0 & 0 \\
Rash & 1 & 0 & 0 \\
\hline
\end{tabular}

reported by previous studies. Furthermore, the median PFS was 8.6 months and the median OS was 16.1 months.

The PFS was similar to that reported by previous studies $(8,9)$. The OS was different, although it was similar to that reported for radiation alone (16.9 months) in the JCOG0301 trial (8).

Concurrent chemoradiotherapy is considered to be the standard treatment for locally advanced NSCLC in selected patients with a good PS (14). Despite the high frequency of NSCLC in the elderly population, elderly patients are frequently underrepresented in clinical trials evaluating chemoradiotherapy $(15,16)$. This is due to elderly patients generally being incapable of tolerating the treatment-related toxicity. In addition, the expectations for long-term benefits may be limited on the part of physicians, as well as on the part of the patients or their families. Therefore, the number of clinical trials designed to specifically study the treatment of elderly patients with stage III NSCLC is limited $(17,18)$.

The results of age-based retrospective subgroup analyses of randomized phase III trials that evaluated concurrent chemoradiotherapy were previously reported by 5 studies (18-22). Those studies reported that healthy older adults with locally advanced NSCLC benefitted from concurrent chemoradiotherapy similar to younger patients, but experienced higher rates of hospitalization and toxicity. A previous meta-analysis 
Table V. Results of previous studies and the present study.

\begin{tabular}{lccccc}
\hline Studies & $\begin{array}{c}\text { Patient age, years } \\
\text { median (range) }\end{array}$ & $\begin{array}{c}\text { ORR } \\
(\%)\end{array}$ & $\begin{array}{c}\text { PFS } \\
(\text { months })\end{array}$ & $\begin{array}{c}\text { OS } \\
\text { (months) }\end{array}$ & $\begin{array}{c}\text { Grade 3 or worse AEs (\%) } \\
\text { (neutropenia/pneumonitis/esophagitis) }\end{array} \quad$ Refs. \\
\hline $\begin{array}{l}\text { WJTOG0105 weekly } \\
\text { CBDCA+PAC arm }\end{array}$ & $63(38-74)$ & 63.3 & 9.5 & 22.0 & $61.6 / 4.1 / 8.2$ \\
$\begin{array}{l}\text { JCOG0301 } \\
\text { chemoradiotherapy arm }\end{array}$ & $77(71-89)$ & 51.5 & 8.9 & 22.4 & $57.2 / 1.0 / 1.0$ \\
$\begin{array}{l}\text { Present study } \\
78(75-86)\end{array}$ & 90.0 & 8.6 & 16.1 & $20 / 0 / 0$ \\
\hline
\end{tabular}

ORR, objective response rate; PFS, progression-free survival; OS, overall survival; AEs, adverse events; WJTOG, West Japan Thoracic Oncology Group; CBDCA, carboplatin; PAC, paclitaxel; JCOG, Japan Clinical Oncology Group.

A

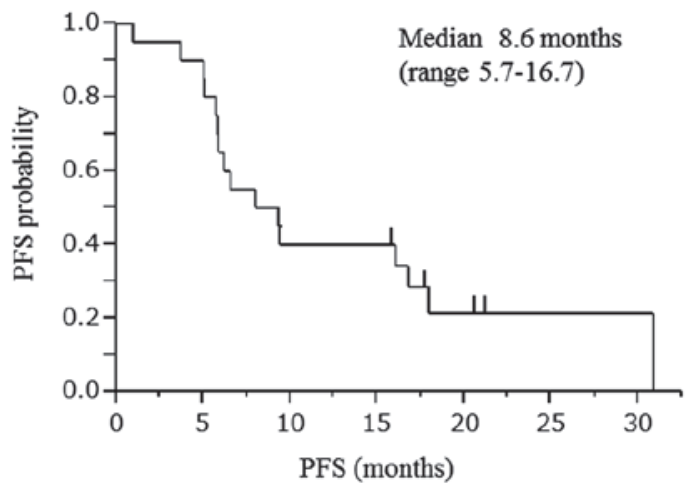

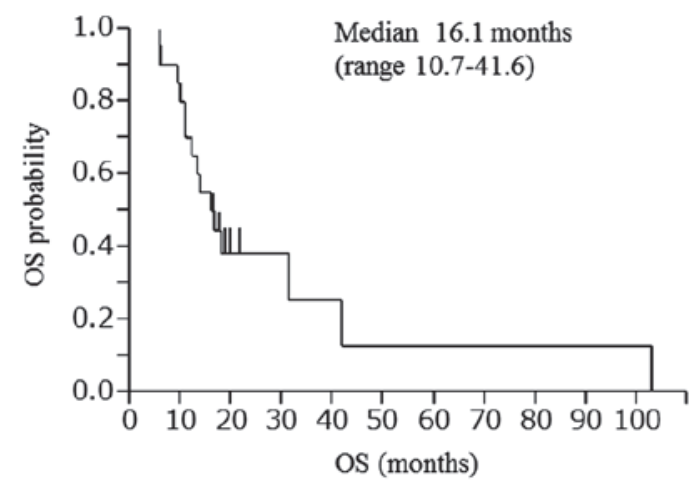

Figure 2. Kaplan-Meier curves for (A) progression-free survival (PFS) and (B) overall survival (OS).

reported that the benefit of concomitant chemotherapy appeared to be greater in elderly compared to that in younger patients (23).

In the JCOG0301 study, Atagi et al (8), reported that the median OS for the chemoradiotherapy (TRT with daily low-dose carboplatin) and radiation therapy alone groups were 22.4 and 16.9 months, respectively. Chemoradiotherapy was associated with a significantly longer survival compared to radiation therapy alone. That study was the first to demonstrate that combined chemoradiotherapy may improve the outcome of stage III NSCLC in elderly patients. In the present study, weekly paclitaxel and carboplatin with concurrent TRT did not provide a survival benefit when compared to the results of the JCOG0310 study.

The West Japan Thoracic Oncology Group (WJTOG0105), in a phase III trial, evaluated concurrent chemoradiotherapy using second-generation regimens at full doses or third-generation regimens at reduced doses in patients with locally advanced NSCLC aged $<75$ years. The results of that trial demonstrated that treatment with weekly paclitaxel and carboplatin with TRT was equally efficacious and exhibited a more favorable toxicity profile compared to the second-generation regimens (9). The ORR was $63.3 \%$, the median PFS was 9.5 months and the median survival time and 5-year survival rate were 22.0 months and $19.8 \%$, respectively, with the weekly paclitaxel and carboplatin treatment. In the WJTOG0105 study, the status of chemotherapy implementation was reported as $58.5 \%$ of patients who received 6 weekly cycles of chemotherapy during the concurrent phase and $49.7 \%$ of patients who received the two scheduled courses of therapy during the consolidation phase. The incidence of grade 3 or worse hematological toxicity was leukopenia in $66 \%$, neutropenia in $61 \%$ and febrile neutropenia in $10.2 \%$ of the patients. Grade 3 or worse pneumonitis occurred in $4.1 \%$ and esophagitis in $8.2 \%$ of the patients in the weekly paclitaxel and carboplatin group.

The present study revealed a lower incidence of grade 3 or worse hematological toxicity, pneumonitis and esophagitis compared to those reported by the WJTOG0105 study for the weekly paclitaxel and carboplatin group. Although our study exhibited similar chemotherapy implementation, ORR, PFS and milder toxicity, it failed to demonstrate a survival benefit compared to other studies (Table V), indicating that our retrospective and small cohort group was not sufficient to provide statistically valid results. Concurrent TRT and chemotherapy with weekly paclitaxel and carboplatin is potentially an effective and feasible treatment for elderly patients; however, our retrospective cohort was insufficient to demonstrate efficacy. Future larger and well-designed prospective clinical trials are required to verify survival benefits.

The limitations of our study lie with its retrospective nature, the small cohort and the lack of a QOL assessment. The intervals between the evaluations of the lesions in this study were not as accurate as those in a prospective study. In addition, the severity of the adverse events may have been underestimated in the present study due to its retrospective nature. The patients 
were hospitalized during most of the treatment period and the toxicity data were recorded in detail in the patients' medical records. The sample size in the present study was limited; therefore, it was difficult to reach a definitive conclusion. However, the collection of data on a large number of patients with locally advanced NSCLC, aged $\geq 75$ years, treated with chemoradiotherapy, is difficult. This retrospective study may therefore be useful for physicians to determine the optimal treatment strategy for patients aged $\geq 75$ years with locally advanced NSCLC.

Previous studies demonstrated that QOL is an important prognostic factor in patients with lung cancer (24-31). One randomized phase III study reported QOL as a prognostic factor for long-term survival among patients with locally advanced NSCLC treated with chemoradiotherapy (32). However, in elderly NSCLC patients, the adverse effects of chemoradiotherapy and their negative effect on the QOL have not been determined. QOL assessment is required for future clinical trials of chemoradiotherapy in elderly patients.

In conclusion, weekly paclitaxel and carboplatin with concurrent TRT failed to demonstrate a clinically significant survival benefit in elderly patients with locally advanced NSCLC. However, this regimen had a tolerable safety profile and an improved objective response. Therefore, it is suggested that this regimen may be suitable for elderly patients; however, further prospective clinical trials are required to evaluate the true efficacy and safety of weekly paclitaxel and carboplatin with concurrent TRT for the treatment of elderly patients with NSCLC.

\section{References}

1. Siegel R, Naishadham D and Jemal A: Cancer statistics, 2013 CA Cancer J Clin 63: 11-30, 2013.

2. National Cancer Institute: SEER Stat fact sheets: Lung and bronchus cancer. http://seer.cancer.gov/statfacts/html/lungb.html Accessed August 12, 2013.

3. Furuse K, Fukuoka M, Kawahara M, et al: Phase III study of concurrent versus sequential thoracic radiotherapy in combination with mitomycin, vindesine, and cisplatin in unresectable stage III non-small-cell lung cancer. J Clin Oncol 17: 2692-2699, 1999.

4. Curran WJ Jr, Paulus R, Langer CJ, et al: Sequential vs. concurrent chemoradiation for stage III non-small cell lung cancer: randomized phase III trial RTOG 9410. J Natl Cancer Inst 103: 1452-1460, 2011.

5. Dillman RO, Herndon J, Seagren SL, Eaton WL Jr and Green MR: Improved survival in stage III non-small-cell lung cancer: seven-year follow-up of cancer and leukemia group B (CALGB) 8433 trial. J Natl Cancer Inst 88: 1210-1215, 1996

6. Sause W, Kolesar P, Taylor S IV, et al: Final results of phase III trial in regionally advanced unresectable non-small cell lung cancer: Radiation Therapy Oncology Group, Eastern Cooperative Oncology Group, and Southwest Oncology Group. Chest 117: $358-364,2000$

7. Edelman MJ, Tang M, Gardner JF, Mullins CD, Seal B and Davidoff AJ: Therapy (Tx) of locally advanced (LA) NSCLC in the elderly: Analysis of 6,325 patients from surveillance, epidemiology and end results (SEER)-Medicare. J Clin Oncol 26 (Suppl 15): 7549, 2008.

8. Atagi S, Kawahara M, Yokoyama A, et al; Japan Clinical Oncology Group Lung Cancer Study Group: Thoracic radiotherapy with or without daily low-dose carboplatin in elderly patients with non-small-cell lung cancer: a randomised, controlled, phase 3 trial by the Japan Clinical Oncology Group (JCOG0301). Lancet Oncol 13: 671-678, 2012

9. Yamamoto N, Nakagawa K, Nishimura Y, et al: Phase III study comparing second- and third-generation regimens with concurrent thoracic radiotherapy in patients with unresectable stage III non-small-cell lung cancer: West Japan Thoracic Oncology Group WJTOG0105. J Clin Oncol 28: 3739-3745, 2010
10. Goldstraw P, Crowley J, Chansky K, et al; International Association for the Study of Lung Cancer International Staging Committee; Participating Institutions: The IASLC Lung Cancer Staging Project: proposals for the revision of the TNM stage groupings in the forthcoming (seventh) edition of the TNM Classification of malignant tumours. J Thorac Oncol 2: 706-714, 2007.

11. Oken MM, Creech RH, Tormey DC, et al: Toxicity and response criteria of the Eastern Cooperative Oncology Group. Am J Clin Oncol 5: 649-655, 1982

12. Eisenhauer EA, Therasse P, Bogaerts J, et al: New response evaluation criteria in solid tumours: revised RECIST guideline (version 1.1). Eur J Cancer 45: 228-247, 2009.

13. National Cancer Institute: Common Toxicity Criteria (NCI-CTC) version 4.0.3. http://evs.nci.nih.gov/ftp1/CTCAE/ CTCAE 4.03 2010-06-14_QuickReference_5x7.pdf. Accessed August 13, 2013.

14. National Comprehensive Cancer Network: Clinical practice guidelines in oncology: non-small cell lung cancer, NCCN guidelines version 2 (2013). http://www.nccn.org/professionals/ physician_gls/pdf/nscl.pdf Accessed August 13, 2013.

15. Hutchins LF, Unger JM, Crowley JJ, Coltman CA Jr and Albain KS: Underrepresentation of patients 65 years of age or older in cancer-treatment trials. N Engl J Med 341: 2061-2067, 1999.

16. De Ruysscher D, Botterweck A, Dirx M, et al: Eligibility for concurrent chemotherapy and radiotherapy of locally advanced lung cancer patients: a prospective, population-based study. Ann Oncol 20: 98-102, 2009.

17. Socinski MA, Zhang C, Herndon JE II, et al: Combined modality trials of the Cancer and Leukemia Group B in stage III non-small-cell lung cancer: analysis of factors influencing survival and toxicity. Ann Oncol 15: 1033-1041, 2004.

18. Rocha Lima CM, Herndon JE II, Kosty M, Clamon G and Green MR: Therapy choices among older patients with lung carcinoma: an evaluation of two trials of the Cancer and Leukemia Group B. Cancer 94: 181-187, 2002.

19. Langer CJ, Hsu C, Curran WJ, et al: Elderly patients (pts) with locally advanced non-small cell lung cancer (LA-NSCLC) benefit from combined modality therapy: Secondary analysis of radiation therapy oncology group (RTOG) 94-10. Proceedings of the Annual Meeting of the American Society for Clinical Oncology 21 (abstract 1193): 299a, 2002.

20. Schild SE, Stella PJ, Geyer SM, et al: The outcome of combinedmodality therapy for stage III non-small-cell lung cancer in the elderly. J Clin Oncol 21: 3201-3206, 2003.

21. Jalal SI, Riggs HD, Melnyk A, et al: Updated survival and outcomes for older adults with inoperable stage III non-small-cell lung cancer treated with cisplatin, etoposide, and concurrent chest radiation with or without consolidation docetaxel: analysis of a phase III trial from the Hoosier Oncology Group (HOG) and US Oncology. Ann Oncol 23: 1730-1738, 2012

22. Sgroi MM, Neubauer M, Ansari R, et al: An analysis of elderly patients (pts) treated on a phase III trial of cisplatin $(\mathrm{P})$ plus etoposide (E) with concurrent radiotherapy (CRT) followed by docetaxel (D) vs observation (O) in pts with stage III non small cell lung cancer (NSCLC). J Clin Oncol 25 (Suppl 18): 9037, 2007.

23. Aupérin A, Le Péchoux C, Pignon JP, et al; Meta-Analysis of Cisplatin/carboplatin based Concomitant Chemotherapy in non-small cell Lung Cancer (MAC3-LC) Group: Concomitant radiochemotherapy based on platin compounds in patients with locally advanced non-small cell lung cancer (NSCLC): a meta-analysis of individual data from 1764 patients. Ann Oncol 17: 473-483, 2006.

24. Montazeri A, Milroy R, Hole D, McEwen J and Gillis CR: Quality of life in lung cancer patients: as an important prognostic factor. Lung Cancer 31: 233-240, 2001.

25. Langendijk H, Aaronson NK, de Jong JM, ten Velde GP, Muller MJ and Wouters M: The prognostic impact of quality of life assessed with the EORTC QLQ-C30 in inoperable non-small cell lung carcinoma treated with radiotherapy. Radiother Oncol 55: 19-25, 2000.

26. Efficace F, Bottomley A, Smit EF, et al: Is a patient's self-reported health-related quality of life a prognostic factor for survival in non-small-cell lung cancer patients? A multivariate analysis of prognostic factors of EORTC study 08975. Ann Oncol 17: 1698-1704, 2006.

27. Maione P, Perrone F, Gallo C, et al: Pretreatment quality of life and functional status assessment significantly predict survival of elderly patients with advanced non-small-cell lung cancer receiving chemotherapy: a prognostic analysis of the multicenter Italian lung cancer in the elderly study. J Clin Oncol 23: 6865-6872, 2005. 
28. Herndon JE II, Fleishman S, Kornblith AB, Kosty M, Green MR and Holland J: Is quality of life predictive of the survival of patients with advanced nonsmall cell lung carcinoma? Cancer 85: 333-340, 1999.

29. Brown J, Thorpe H, Napp V, et al: Assessment of quality of life in the supportive care setting of the big lung trial in non-small-cell lung cancer. J Clin Oncol 23: 7417-7427, 2005.

30. Ganz PA, Lee JJ and Siau J: Quality of life assessment. An independent prognostic variable for survival in lung cancer. Cancer 67: 3131-3135, 1991.
31. Eton DT, Fairclough DL, Cella D, Yount SE, Bonomi P and Johnson DH; Eastern Cooperative Oncology Group: Early change in patient-reported health during lung cancer chemotherapy predicts clinical outcomes beyond those predicted by baseline report: results from Eastern Cooperative Oncology Group Study 5592. J Clin Oncol 21: 1536-1543, 2003.

32. Movsas B, Scott C, Langer C, et al: Randomized trial of amifostine in locally advanced non-small-cell lung cancer patients receiving chemotherapy and hyperfractionated radiation: Radiation Therapy Oncology Group Trial 98-01. J Clin Oncol 23: 2145-2154, 2005. 